\title{
Dynamic conceptual blending analysis to model student reasoning processes while integrating mathematics and physics: A case study in the context of the heat equation
}

\author{
Sofie Van den Eynde $\odot,{ }^{1,2, *}$ Benjamin P. Schermerhorn, ${ }^{3}$ Johan Deprez $\odot,{ }^{4}$ \\ Martin Goedhart, ${ }^{2}$ John R. Thompson $\odot,{ }^{5}$ and Mieke De Cock ${ }^{1}$ \\ ${ }^{1}$ Department of Physics and Astronomy \& LESEC, KU Leuven, \\ Celestijnenlaan 200c, 3001 Leuven, Belgium \\ ${ }^{2}$ Institute for Science Education and Communication, University of Groningen, \\ Nijenborgh 9, 9747 AG Groningen, Netherlands \\ ${ }^{3}$ Department of Physics, California State University, Fullerton, Fullerton, California 92831, USA \\ ${ }^{4}$ Department of Mathematics \& LESEC, KU Leuven, Celestijnenlaan 200c, 3001 Leuven, Belgium \\ ${ }^{5}$ Department of Physics and Astronomy \& Maine Center for Research in STEM Education, \\ University of Maine, Orono, Maine 04469, USA
}

(Received 18 December 2019; accepted 18 February 2020; published 27 March 2020)

\begin{abstract}
In recent years, there has been an increased interest in conceptual blending in physics and mathematics education research as a theoretical framework to study student reasoning. In this paper, we adapt the conceptual blending framework to construct a blending diagram that not only captures the product but also the process of student reasoning when they interpret a mathematical description of a physical system. We describe how to construct a dynamic blending diagram (DBD) and illustrate this using two cases from an interview study. In the interview, we asked pairs of undergraduate physics and mathematics students about the physical meaning of boundary conditions for the heat equation. The selected examples show different aspects of the DBD as an analysis method. We show that by using a DBD, we can judge the degree to which students integrate their understandings of mathematics and physics. The DBD also enables the reader to follow the line of reasoning of the students. Moreover, a DBD can be used to diagnose difficulties in student reasoning.
\end{abstract}

DOI: 10.1103/PhysRevPhysEducRes.16.010114

\section{INTRODUCTION}

Interpreting the way students use and understand the mathematics used in physics is an important and central research theme in physics education research (PER) (e.g., Refs. [1-9]). Proficiency in mathematics is required to describe and understand physical phenomena, and being able to combine the different fields is a prerequisite to become more proficient in physics. Understanding an equation in physics goes beyond connecting the symbols to physical quantities and being able to perform calculations and operations with that equation. It also involves being able to connect mathematical knowledge and representations to physical meaning and integrating an equation with its implications in the physical world [10]. Parsing students' mathematical and physical understanding has proven challenging as the use of mathematics in physics

\footnotetext{
*sofie.vandeneynde@kuleuven.be
}

Published by the American Physical Society under the terms of the Creative Commons Attribution 4.0 International license. Further distribution of this work must maintain attribution to the author(s) and the published article's title, journal citation, and DOI. is more than just the sum of both parts. Different authors have adopted the conceptual blending perspective [11] to describe student understanding at the mathematics and physics interface $[4,12,13]$. Huynh and Sayre [14] state that "the effective use of mathematics in making sense of the physical world involves blending reciprocally between mathematics and physics contexts rather than just applying mathematics to physics."

In this study, we focus on student reasoning about boundary conditions for a partial differential equation, more specifically the heat equation. In a physical system, the boundary conditions define the conditions physical quantities must satisfy at the boundary of the system. Boundary conditions are particularly critical because they are necessary to reduce general and abstract mathematical expressions to physically meaningful solutions that have descriptive and predictive power within a particular physical system [15]. Therefore, this is a promising context to investigate the role of mathematics and physics understanding in student reasoning.

In a previous study [16], we investigated student difficulties with boundary conditions in the context of particle diffusion, which is mathematically described in the same way as heat flow. We identified difficulties related to 
mathematics, physics, and the connection between both. This has been an important first step in investigating student understanding of boundary conditions. However, we need more information about the way our students combine mathematics and physics while reasoning about boundary conditions in order to develop effective teaching and learning materials that scaffold meaningful blending. In this study, we extend the focus on the reasoning process in the conceptual blending model.

We start with a brief review of the physical context we focus on in this study, one-dimensional systems in which heat flow can be described by the heat equation. Then we discuss the original conceptual blending framework in Sec. I B. We highlight relevant adaptations of the framework in physics (PER) and mathematics education research (MER) (Sec. IC), which brings us to the specific aims and research questions of this study (Sec. ID). In the methodology section (Sec. II) we discuss the participants, the interview content, and protocol. We give a detailed description of the data analysis method in Sec. III. Using two case studies, we illustrate the construction of dynamic blending diagrams (Sec. IV). We end with a discussion (Sec. V) containing limitations and suggestions for future research.

\section{A. Heat flow in one-dimensional systems}

In this study, we confronted students with physical situations that can be described by the one-dimensional heat equation. We briefly review this partial differential equation and how it describes heat transfer in a system.

If we consider a one-dimensional system of length $L$ in which the temperature at position $x$ and time $t$ is described by $u(x, t)$, the evolution of the temperature in the system can be modeled by the following partial differential equation:

$$
\frac{\partial u}{\partial t}(x, t)=\alpha \frac{\partial^{2} u}{\partial x^{2}}(x, t),
$$

for $0<x<L$ and $0<t<\infty$. In this equation, $\alpha$ is the thermal diffusivity, a measure of the rate at which heat can spread in a specific medium.

The equation relates the quantities $\partial u / \partial t(x, t)$, the rate of change in temperature with respect to time, and $\partial^{2} u / \partial x^{2}(x, t)$, the concavity of the temperature distribution $u(x, t)$, which essentially compares the temperature at one point to the temperature at neighboring points [17].

To fully describe the system, the initial temperature distribution $u(x, t=0)$ and the conditions describing what happens at the boundary of the system are also needed. Boundary conditions refer to the conditions physical quantities must satisfy at the boundary of the system at all times. In the case studies selected here, we limit ourselves to boundary conditions of the form $u(x, t)=c$ at $x=0$ or $x=L$ with $c$ being a constant. This type of boundary condition specifies the value of the temperature at the boundary.

In the case studies, a crucial element in the reasoning of the students is the mathematical description of the heat flow. This can be explained through the law of heat conduction, also known as Fourier's law, states that the rate of heat transfer through a material is proportional to the negative of the gradient in the temperature. In the onedimensional differential form, this can be written as

$$
q\left(x_{0}, t_{0}\right)=-k A \frac{\partial u}{\partial x}\left(x_{0}, t_{0}\right),
$$

where $q(x, t)$ is the local heat flux density, which is the amount of heat that flows through a unit area per unit time, and $k$ is the thermal conductivity, which is a measure of how well the material conducts heat [17]. This shows that the derivative of temperature with respect to position, which expresses the local temperature difference, is a measure for the heat transferred. Fourier's law states that if $\partial u / \partial x\left(x_{0}, t\right)<0$, heat will flow from left to right; and if $\partial u / \partial x\left(x_{0}, t\right)>0$, then the flow of heat through point $x_{0}$ will be from right to left, because heat always flows from high to low temperatures.

\section{B. The conceptual blending framework}

Conceptual blending, or sometimes called mental space integration, was originally introduced by Fauconnier and Turner [18] to model how people create new meaning in linguistic contexts by selectively combining information from previous experiences.

A general schematic representation of the conceptual blending framework is shown in Fig. 1. In its basic form, a conceptual blending network consists of four connected mental spaces: two partially matched input spaces, a generic space, and the blended space. Generally, a mental space is comprised of conceptual packets or knowledge elements that tend to be activated together, and has an

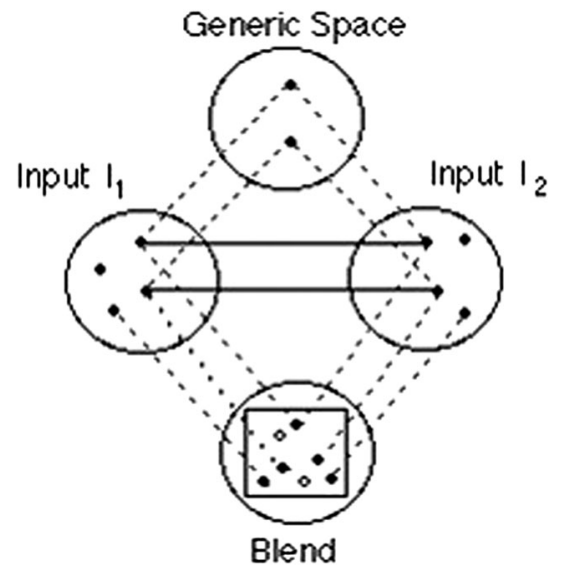

FIG. 1. Schematic representation of a blending diagram from the original work of Fauconnier and Turner [11]. 
organizing frame that specifies the relationships, or connections among the elements [13]. Input spaces are small self-contained regions of conceptual ideas. The generic space provides the underlying structure to the input spaces, identifying commonalities in content and structure [11].

Blended spaces are constructed through selective projection from the inputs. Fauconnier and Turner distinguish three mechanisms to form a blend: composition (blending can compose elements from the input spaces to provide relations that do not exist in the separate input spaces), completion (adding new elements based on background models that are brought into the blend unconsciously), and elaboration (treating the blend as a simulation and "running" it imaginatively, which creates new insights) [11]. The blend has emergent dynamics. It can be "run" while its connections to the other spaces remain in place.

\section{Conceptual blending to describe students' use of mathematics in physics}

Considering that mathematics serves as a way to carry and relay information about physical contexts, the conceptual blending framework provides a means to explore student understanding as they connect mathematics and physics concepts. In PER, the conceptual blending framework has repeatedly been used to model the blending of physics and mathematics. An important sign of physics students' progress is combining the symbols and structures of mathematics with their physical knowledge and intuition, enhancing both. New ideas and inferences emerge after this combination. The conceptual blending framework emphasizes both the new combinations of elements and the different ways that combination itself can be constructed [12].

Bing and Redish were the first to introduce the language of conceptual blending as a way to analyze problem solving in physics at the introductory level [12]. They identified two input spaces, called "mathematical machinery" and "physical world" (see Fig. 2). In their adaptation, the role of mathematics was limited to its technical aspect. Hu and Rebello, and Bollen and colleagues extended the adaptation of the blending framework to also consider the conceptual aspect of mathematics, looking at how students blended concepts from electrodynamics with the mathematical concepts of integration [4] and vector differential operators [13]. They identified three input spaces: the symbolic space (abstract mathematical symbols and notations), the math notion space (knowledge about mathematical concepts and notations), and the physics space (physical quantities associated with the object) (see Fig. 3). The addition of the math notion space accounts for the conceptual role of mathematics. Apart from the technical role, where mathematics is seen as a tool to process physics, there is also the structural role, where mathematics is significant to the construction of the physical concept itself [19].

Schermerhorn and Thompson [20] incorporated the symbolic forms framework [21] into the conceptual

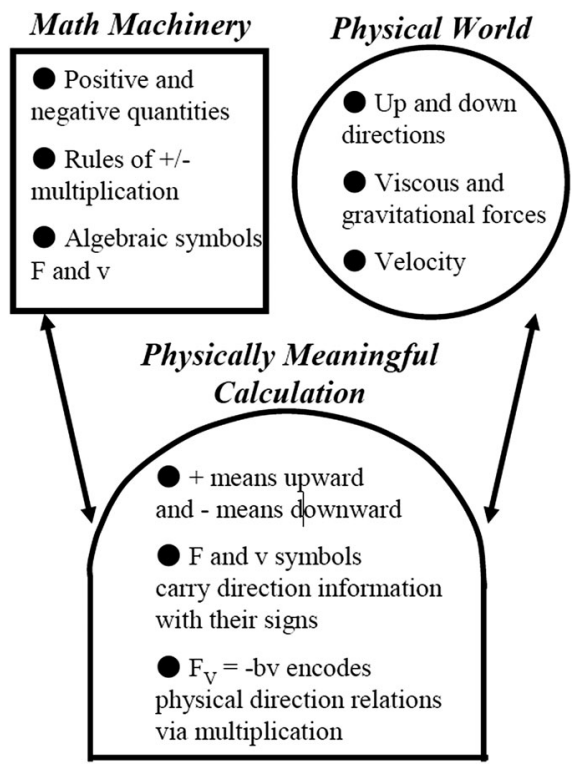

FIG. 2. Example of a blending diagram from the work of Bing and Redish [12].

blending framework to describe students' construction of differential length vectors and extend their model to equation construction and interpretation generally. Their model explicitly connects elements between input spaces, highlighting the mechanism of blending in the construction of equations. The authors incorporated the generic space as an underlying mathematical justification to provide the structure of the blending diagram for this model (Fig. 4). As the mathematical structure (left input space) and contextual information (right input space) are blended, the result is an equation, a collection of symbols and ideas that can only be understood in the light of both input spaces.

In MER, conceptual blending has been used to capture the process of student reasoning. Gerson and Walter [22] used conceptual blending to describe the emergence of students' understanding of calculus concepts during sustained mathematical inquiry. Students visited and revisited

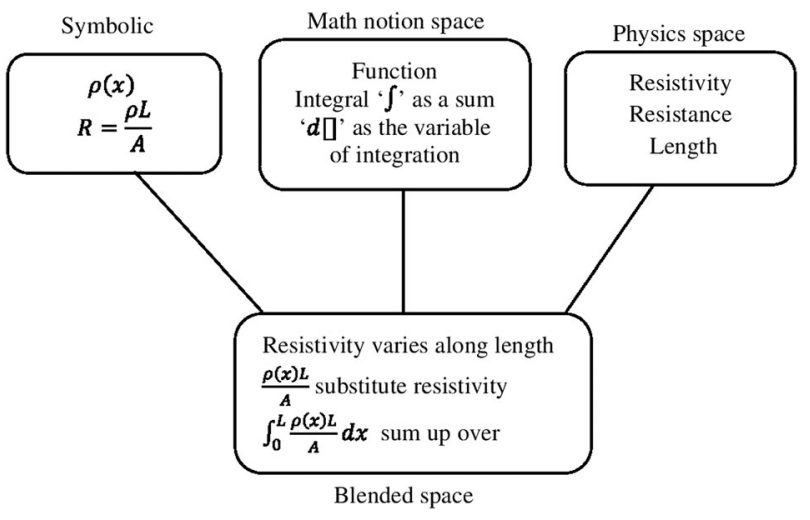

FIG. 3. Example of a blending diagram from the work of $\mathrm{Hu}$ and Rebello [4]. 


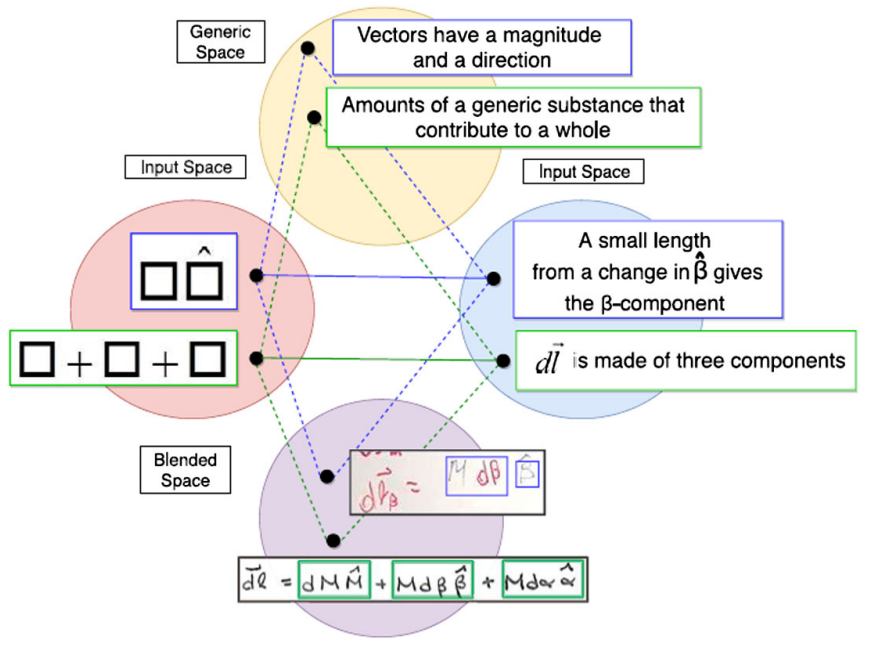

FIG. 4. Blending diagram from Schermerhorn and Thompson [20]. In this example, students' construction of a differential length vector comes from attending to the magnitude and direction of each component and to the sum of components from three different directions. Combining their content knowledge with the mathematical templates, the students write the final expression. The determination of the terms in each magnitude (not shown here) is also the result of blending different symbolic forms with content knowledge.

important calculus content during a teaching experiment, but not necessarily along a path predetermined by the instructors. Key episodes were coded for content and connections, and analyzed by creating maps of conceptual blends. An example of such a map, which is a representation of the students' connected understandings, can be found in Fig. 5. In this example, the students are answering a question about the amount of water in a reservoir. The researchers identified two input spaces: the ideas concerning in flow and out flow, and one about the context of water in a reservoir. These two input spaces are combined into a blended space where the students discuss the quantity of water in the reservoir. Two aspects of this work

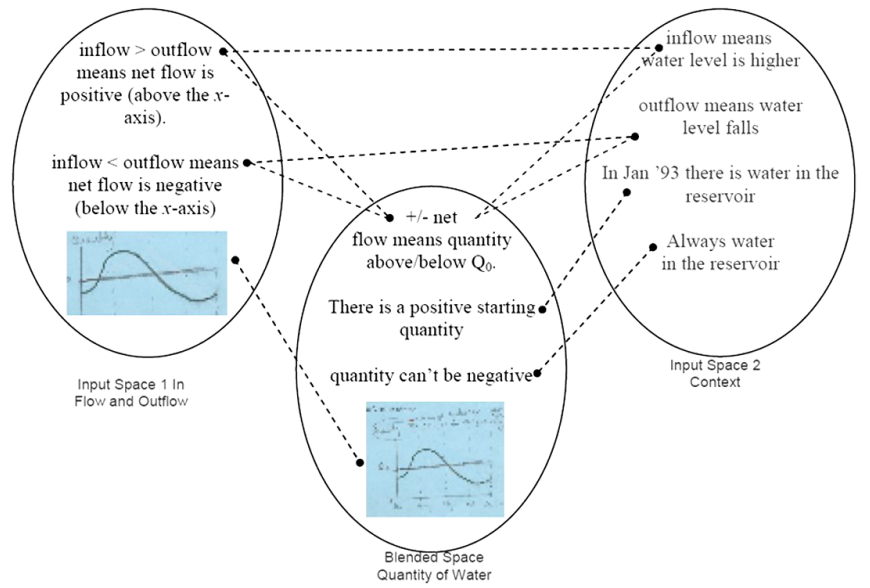

FIG. 5. Blending diagram from the work of Gerson and Walter [22]. are of particular interest to our own project. First, like Schermerhorn and Thompson, these authors identified individual connections between elements in the mental spaces, instead of just connecting the entire spaces. They state that analyzing both the content and the connections students make among content, context and previous knowledge, gives a richer picture of the emergent meaning students are creating as they explore meaningful mathematics tasks. Second, graphs received explicit attention in this work. The abstract graph was placed in the abstract flow space, but once this graph was used to explicitly discuss the water in the reservoir, it became a blended element.

Zandieh, Roh, and Knapp [23] show in their work how conceptual blending can be used as a methodological tool for organizing the story line of the evolution of student thinking. They investigated how small groups of students work on a mathematical proof. They do not use one blending diagram to represent the whole process, but divide the reasoning process in different episodes. In each episode, a primary blend is constructed by the group. However, they also distinguish a secondary blend. This secondary blend accounts for individual students' contrasting ideas that might alter the primary blend in the next episode.

In most of the PER work, the focus lies on the product of the reasoning, and the blending model is used to describe what elements from both disciplines are combined. The process of students' reasoning, however, is not captured. To scaffold the learning of meaningful blending of mathematics and physics, insight into the process itself is needed. In this paper we therefore propose an adaptation of the model to analyze how students integrate the two disciplines in their reasoning.

\section{Research questions}

The general aim of this study was to adapt the conceptual blending framework as used in PER and MER to not only capture the product but also the process of student reasoning when combining physics and mathematics. During data analysis, this aim resulted in the following research questions:

1. How are the input and blended spaces defined in the context of investigating the role of mathematics and physics in student reasoning about boundary conditions for the heat equation?

2. How can we visualize the students' reasoning process in a dynamic blending diagram?

(a) How can we visualize concepts and ideas that students use while combining mathematics and physics in the context of the heat equation?

(b) How can we visualize the order in which students use and connect these concepts and ideas?

(c) How can we visualize the connections students make?

3. What are the merits of a dynamic blending diagram as an analysis method for student reasoning? 


\section{METHODS}

To illustrate the development of a dynamic blending diagram (DBD), we select two cases from an interview study. In that study, we investigate student reasoning when combining mathematics and physics in the context of the heat equation. We will later use the analysis method developed in this paper to analyze all the data from that interview study.

In this section, we give an overview of the participants, the context, the setup, and the content of the interviews. Lastly, we explain how to construct a DBD starting from data. In the next section, we will illustrate this method using data from the interview study.

\section{A. Participants and context}

The data presented in this paper are part of a study where we conducted task-based, think-aloud interviews with pairs of students to investigate their understanding of boundary conditions in the context of the heat equation. The participating students were second-year physics and mathematics undergraduate students studying at KU Leuven (Belgium). Some weeks before the interview, they finished a course on differential equations, taught in Dutch, which entailed a chapter in which the heat equation was discussed in depth, i.e., the derivation of the heat equation, boundary conditions, physical systems described by this equation, and the algorithmic technique of separation of variables to solve the equation.

In our previous study [16], we observed that individual students had many difficulties reasoning about boundary conditions for the diffusion equation and were often not able to answer the interview questions. This made it difficult to investigate their reasoning. Therefore, by letting students work in pairs, we hoped that they would be able to help each other. A second advantage is that by letting students work in pairs, they naturally question each other, which forces students to be more explicit about their reasoning process.

\section{B. Interview content}

We designed interview problems to elicit explicitly how students blend mathematics and physics concepts while answering the questions. The whole interview contained five problems. However, in this paper we focus on student answers to two of these tasks in order to explain the construction of a DBD (see Figs. 6 and 7). In formulating the interview tasks, we used the course notes and notations therein as a guideline.

The students start from a mathematical description (consisting of a partial differential equation, an initial condition, and boundary conditions) and are asked to reason about the physical meaning. Each task consists of three questions. In the first question (I), we want the students to translate the boundary conditions into physical conditions for the boundary of the system. In this study, we focus on boundary conditions of the form $u(x, t)=a$ with $a$ being constant, which specify the temperature at the boundary for all times. In the second and third questions (II and III), we want the students to reason about the evolution of the system in a qualitative way, without calculating.

\section{Interview protocol}

Each interview lasted approximately one hour, during which the participants were encouraged to explicitly discuss and think aloud as they worked through the tasks. The interviewer was the first author of the paper, and was not involved in the course on partial differential equations. The interviews were conducted using a smart pen, which audio recorded the conversations and kept track of the students' notes, drawings, and calculations. The interviews were also videotaped as a backup for the smart pen.

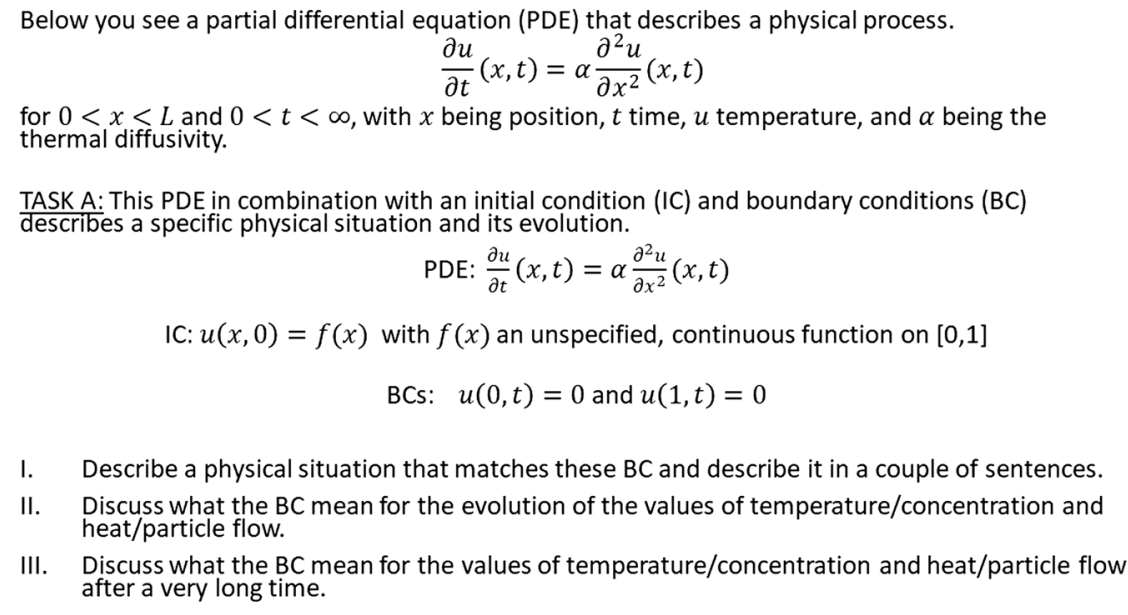

I. Describe a physical situation that matches these $B C$ and describe it in a couple of sentences.

II. Discuss what the $\mathrm{BC}$ mean for the evolution of the values of temperature/concentration and heat/particle flow.

III. Discuss what the $\mathrm{BC}$ mean for the values of temperature/concentration and heat/particle flow after a very long time.

FIG. 6. Interview question: Task A. 
TASK B:

$$
\begin{gathered}
\text { PDE: } \frac{\partial u}{\partial t}(x, t)=\alpha \frac{\partial^{2} u}{\partial x^{2}}(x, t) \\
\text { IC: } u(x, 0)=f(x) \text { with } f(x) \text { an unspecified, continuous function on }[0,10] \\
\text { BCs: } u(0, t)=0 \text { and } u(10, t)=50
\end{gathered}
$$

I. Describe a physical situation that matches these $B C$ and describe it in a couple of sentences.

II. Discuss what the $\mathrm{BC}$ mean for the evolution of the values of temperature/concentration and heat/particle flow.

III. Discuss what the $\mathrm{BC}$ mean for the values of temperature/concentration and heat/particle flow after a very long time.

FIG. 7. Interview question: Task B.

The interview questions were written in English but the students could respond in Dutch. The English interview questions were no problem for the students as they were used to English textbooks and lectures. In case they did not understand the question, the interviewer provided clarity. Data were translated afterwards to English by the interviewer.

At the start of the interview, the interviewer informed the students about the subject and purpose of the study. The interviewer also emphasized that she would not give feedback about the correctness of responses during the interview. After this introduction, the students signed an informed consent form. Generally, the interviewer did not interfere, except for prompts to think aloud and requests for further explanation of what the students did and why. After the interview, the students had the chance to discuss their answers and the aim of the research project in an informal way.

\section{DATA ANALYSIS}

\section{A. Constructing a dynamic blending diagram}

To describe the process of blending mathematics and physics, we construct a dynamic blending diagram. In a first step, the interviews were transcribed verbatim and drawings, calculations, and notes from students were added to the transcripts. The students' names were replaced by pseudonyms to guarantee anonymity.

Based on the transcripts, the DBD is constructed by taking four steps. A schematic overview of these steps is shown in Fig. 8.

1. Elements are identified from the transcript. An element is defined as a new step, idea, concept, or phrase in the reasoning. When a student exactly repeats an idea that has been used before, it is not identified as a new element. We copy the elements from the transcript as literally as possible to minimize bias and interpretations. In order to give a complete picture, all elements that are present in the reasoning are included in the DBD. The elements that are stated in the problem, in our case the partial differential equation, initial condition, and boundary conditions, can also be part of the DBD. It is therefore important that the moments where the students are referring to these elements are included in the transcript. A color code distinguishes between the different types of elements: incorrect elements are represented in a black box with white letters, elements that are given in the problem statement are represented in a gray box, and all other elements are represented in a white box in the DBD. This color code enables the reader to distinguish between elements that students created themselves (white) and elements that were given, but actively used by the students (gray). The black boxes indicate that students are having difficulties.

2. To represent the order of the reasoning steps, the elements are numbered in order of appearance in the transcript.

3. Each element is assigned to a mental space. We identify three spaces in our analysis: a physics space, a mathematics space, and a blended space. The characterization can be found in Table I. We consider each mental space as a shared space for both students. In their investigations of the cognitive processes of small groups in a classroom setting, Megowan and Zandieh [24] state that a group of students is a legitimate unit of analysis when

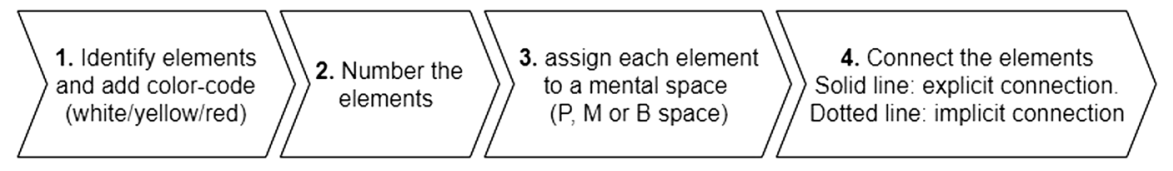

FIG. 8. Schematic overview of the necessary steps to construct a DBD. 
TABLE I. Definitions of the different mental spaces used in this study.

\begin{tabular}{|c|c|}
\hline Space & Definition \\
\hline $\begin{array}{l}\text { Mathematics } \\
\text { space }\end{array}$ & $\begin{array}{l}\text { The mathematics space ( } M \text { space) contains all elements related to mathematical knowledge, without any new physical } \\
\text { input (i.e., using only physical input that has already been mentioned before in the reasoning process). This broadly } \\
\text { entails mathematical concepts, functions, and operations such as derivatives. This also entails graphs and equations } \\
\text { when they are used in an abstract, mathematical way without referring explicitly to a particular physical situation. }\end{array}$ \\
\hline Physics space & $\begin{array}{l}\text { The physics space ( } P \text { space) contains all elements related to physics knowledge, without any new mathematical input. } \\
\text { There can be a variety of levels: using physics concepts; describing a physical system and process or relation in } \\
\text { words; using experimentally known facts or relations between physical quantities; statements about the nature or } \\
\text { state of the physical system. }\end{array}$ \\
\hline Blended space & $\begin{array}{l}\text { The blended space ( } B \text { space) contains elements that combine mathematics and physics ideas. Additionally, equations } \\
\text { and graphs are also categorized as part of the blended space when they connect physical and mathematical meaning. } \\
\text { This means that the boundary conditions given in the problem statement will be placed here if students give a } \\
\text { physical interpretation of these equations in their answer. }\end{array}$ \\
\hline
\end{tabular}

examining student reasoning. In such a setting, content knowledge can be seen as an emergent property of group interactions.

4. The numbered elements are connected in the DBD to visualize the connections in the students' reasoning. We distinguish between explicit and implicit connections. When students explicitly connect elements in their reasoning, these elements are connected with a solid line. Sometimes the connection is not clear from the transcript alone, but from the course of the interview, there are reasons to assume that the elements are connected. In that case, they are connected with a dotted line.

Using this representation, blending cannot be limited to the blended space alone, but is also visible in the connections between all spaces. The total of the elements in the three mental spaces and the connections between the elements gives an overview of the blending of mathematics and physics.

\section{B. Reliability}

In developing and refining the method of constructing a DBD, the first two authors coded student statements, equations, and sketches as either mathematical, physical, or blended elements. The DBDs were discussed among the remaining authors and refined until agreement on placement of elements and connections between elements. Once the methodology was considered final, two authors independently constructed a DBD starting from transcriptions from three different, randomly selected, segments of the interviews that were not analyzed before (we will refer to them as R1, R2, and R3). We discuss the reliability on four levels: the selection of elements, the categorization of elements, the number of connections and the type of connection. The percentages of agreement for every level are shown in Table II.

As part of the first stage, selection of elements, we listed the identified elements for both authors and checked for agreement. We calculated a percentage of agreement by dividing the number of agreeing elements by the total number of elements identified. The total number of identified elements is provided in Table II. For the second stage, we checked the placement of each element in the $M$, $P$, or blended space. Because the agreement in selection was high, we limited our further analysis to the common elements. We calculated a percentage of agreement by dividing the number of agreeing categorizations by the number of commonly identified elements. Third, we listed all identified connections between the common elements and checked for agreement. Lastly, the type of connection was checked for agreement.

Overall, all percentages of agreement are high (>75\%), which indicates that two independent authors made very similar dynamic blending diagrams from the same data based on the methodology as it is described in this paper.

\section{CONSTRUCTING A DYNAMIC BLENDING DIAGRAM IN PRACTICE: TWO CASE STUDIES}

To demonstrate the construction of a DBD, two case studies are presented that each highlight different aspects of the DBD.

TABLE II. Percentages of agreement between two authors that coded three pieces of data independently (R1, R2, and R3). For each piece of data, we add the amount of identified elements between brackets (\# el.). Reliability is discussed on four levels: the selection of elements, the categorization of elements, the number of connections, and the type of connection.

\begin{tabular}{lcccr}
\hline \hline & Selection & Categorization & Connections & Type \\
\hline R1 (11 el.) & 90.9 & 90 & 80 & 75 \\
R2 (8 el.) & 100 & 100 & 100 & 100 \\
R3 (9 el.) & 78 & 85.7 & 85.7 & 83 \\
\hline \hline
\end{tabular}




\section{A. Case 1}

As a first example, we analyze the answer of two students, David and Evan, to interview task B (see Fig. 7). We selected this case because it shows some instances of blended reasoning, where students combine all three spaces, but also of one-sided reasoning, where students fail to make the connection to the other input spaces.

\section{Question I}

The students start by answering question B-I. The corresponding DBD 1 can be found in Fig. 9. In the transcript, all elements, i.e., new steps, ideas, concepts, or phrases in the reasoning, are marked in bold and they are numbered chronologically. Information about what students are drawing, writing, or pointing at is added between brackets in the transcript.

[David draws a coordinate system for $u(x, t)$ : origin and three axes with labels, but without a graph (1).]

David 21:31 So, the difference in boundary conditions is now that... [writes 'B.C.: $u(0, t)=0$ ' and ' $u(10, t)=$ 50' (2)] Eh yeah, so the start, so the first boundary condition is still the same, but the other one is now something that is not equal to zero.. (3)

Evan 21:55 Yes.

David 21:57 And ehm. And yeah if we are doing it again with heat, so the beginning of the rod is kept at zero and the end of the rod at 50. (4)

Evan 22:15 Yes.

David 22:17 And...

Evan 22:17 [referring to the initial condition (5)] In the beginning it is kind of a weird distribution, possibly a weird distribution (6), from zero to, ehm yeah, to, to, it starts in zero and ends in 50 (7)...

[David draws a straight line at position 10 at u-value 50 and one at position 0 at $u$-value 0 for all times (8) in the coordinate system.]

First, David sketches a 3D coordinate system without doing anything with it. We label this as an element of the blended space because later on David and Evan use the coordinate system to represent the physical situation graphically. Then, the students write down the equations of the boundary conditions given in the problem statement (2, gray box) and compare these equations with the ones

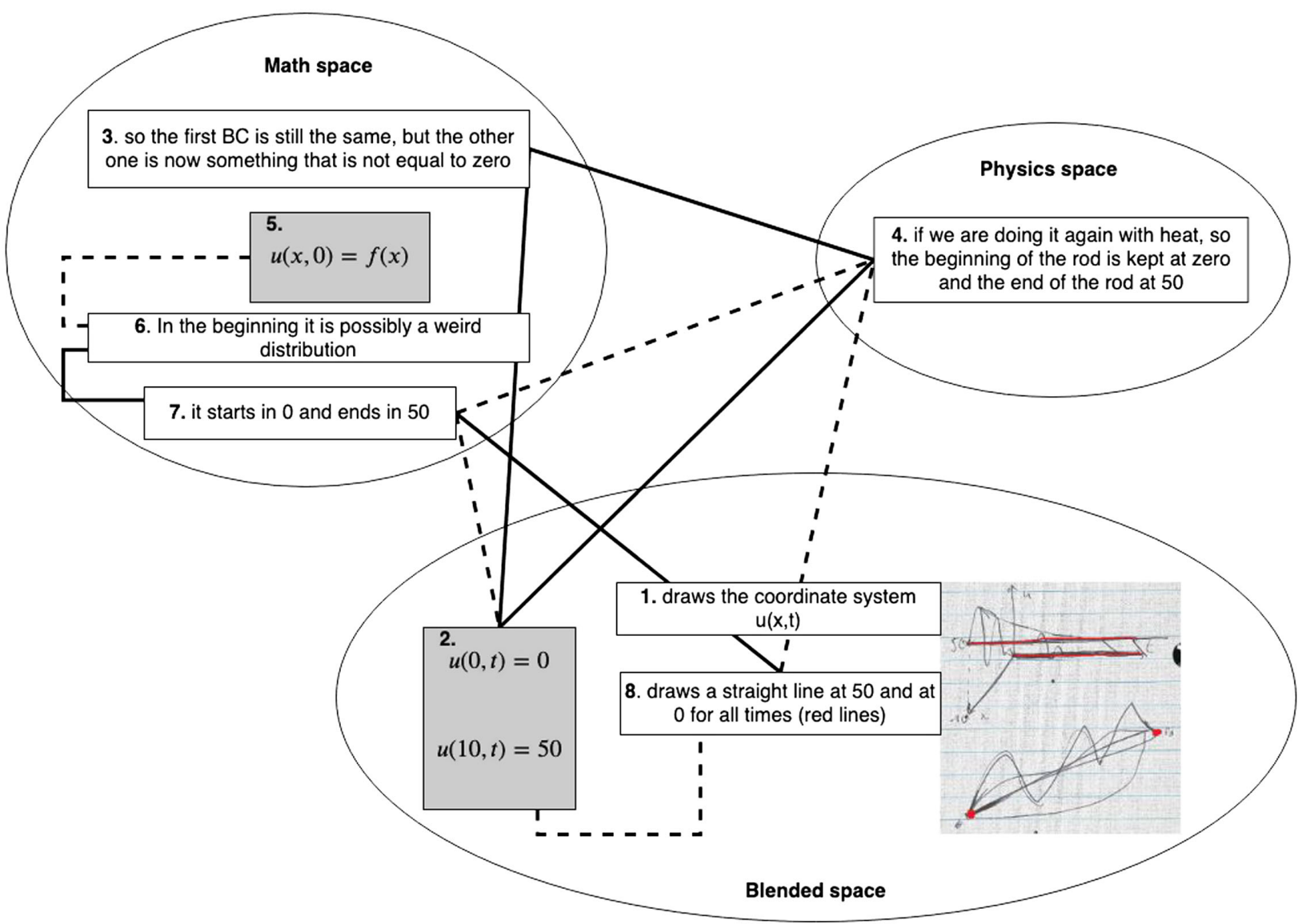

FIG. 9. DBD 1: David and Evan's answer to question B-I. At this point in the transcript, the students only construct parts of the sketch. Top sketch: the coordinate system and the two horizontal lines starting at $(10,50)$ and $(0,0)$. Bottom sketch: the two dots on the sides (at 0 and 50). 
from task A (see Fig. 6). They establish that the first boundary condition is equal to the one in task $\mathrm{A}$, while the right-hand side in the second one is now equal to 50 (so nonzero) (3). Element 2 is categorized as part of the blended space because these are equations that are interpreted physically in this excerpt. We categorize element 3 in the mathematics space because at this point the students are talking about the form of these equations in an abstract way, without explicitly mentioning the physical meaning of this equation. Elements 2 and 3 are connected through a solid line because the two elements are connected by the use of the word "so." Thereafter, the students make the connection to the physics context by referring to the context of heat acknowledging the temperature of the rod being constant at the ends (4) while still comparing with task A. This element is also connected with a solid line to both elements 2 and 3 , because it refers to the physical interpretation of the mathematical equations. This results in the triangle 2-3-4, which shows the mathematical and physical interpretation of the boundary conditions.

Evan interprets the initial condition from the problem statement (5, gray box) and states that in the beginning it is possibly "a weird distribution" (6). Both these elements are categorized as part of the mathematics space because element 5 is an equation without reference to the physical situation it represents and element 6 abstractly describes the shape of the function. Even though the student is explicitly referring to a moment in time (beginning), this is not enough to put this element in the physics space. Time has been established as one of the variables earlier, so this cannot be seen as new physical input in this element. These two elements are connected with a dotted line because the students do not explicitly mention element 5 in words.

Evan then states that the initial function starts in zero and ends in 50 (7). This is placed in the mathematics space because it repeats the values of the boundary conditions at the beginning and end positions without referring to the physical context of temperature and heat. This is explicitly connected to element 6 as this idea follows in the same sentence. It is also implicitly connected to the equations the student is referencing (element 2) and the physical idea of constant temperature (element 4). Finally, David used the abstracted mathematical values as locations on the graph and drew horizontal lines on the set of axes to visualize the fixed values at positions zero and ten; this is element $\mathbf{8}$ and is categorized in the blended space because it graphically represents the boundary conditions and their physical meaning (element 4 , implicit connection). Note that both element 1 and 8 are parts of the graph construction process and so both are placed in the blended space. Elements 7 and 8 are explicitly connected. In element 7 , the "it" refers to the initial distribution (so at $t=0$ ), whereas the lines in element 8 deal with all values of $t$. Therefore, elements 2 and 8 are connected implicitly.

\section{Question II}

The students continue and start discussing the evolution of the system over time. Figure 10 shows DBD 2 for the following piece of transcript.

Evan 22:26 It (the graph) flattens out again, because that, that is just what things do under influence of the heat equation, (1) but how would it look like at the end? Something like eh, straight line or so maybe? (2) I don't really know.

David 22:40 Hm...

Evan 22:40 What makes the most sense?

David 22:43 (referring to the boundary condition at position 10 (3)) Wait, the end should be kept at 50 (4), so there should be some kind of heat source. (5)

Evan 22:51 Yes.

David 22:52 Yes, and if there is a heat source, heat is again flowing over there, to the beginning of the rod (6).(referring to the boundary condition at position 0 (7)) But the beginning of the rod is so cold that it stays zero (8) and so it cancels each other out a bit (9) and I think you will just get a straight line (10).

Evan 23:13 Yes, I also have the feeling it will become a straight line, because ehm, if it is not going to be a straight line, what else...

In element 1, Evan states that because of the effect of the heat equation, there is a tendency for the graph to "flatten out." They seem to combine aspects of mathematics and physics in this element, so it is placed in the blended space. He ends the sentence with the thought that the graph will probably evolve to a straight line (2), which is placed in the mathematics space because it refers to the features of the graphical representation. Elements 1 and 2 are connected explicitly.

Next, the students discuss the physical meaning of the boundary conditions. The placement of element $\mathbf{4}$ could be in the mathematics or the physics space. However, the continuation of the sentence suggests that the students are giving a physical interpretation here of the boundary condition at position 10, hence the placement in the physics space. Element $\mathbf{5}$ is then the physical tie to that; you have to know that it requires heat flow to keep a constant temperature. The boundary condition was given in the problem statement ( 3 , gray box). This boundary condition is placed in the blended space because there is a physical interpretation (elements 4 and 5) tied to this equation. The connection between elements 3 and 4 is implicit because the students do not mention the boundary condition explicitly. However, this connection is important because it indicates blending: the students made a physical interpretation of a mathematical equation.

Based on this, the students start a line of reasoning mainly in the physics space that is internally connected with solid lines. They state that heat has to flow to the beginning of the rod (6, physics space), where the 


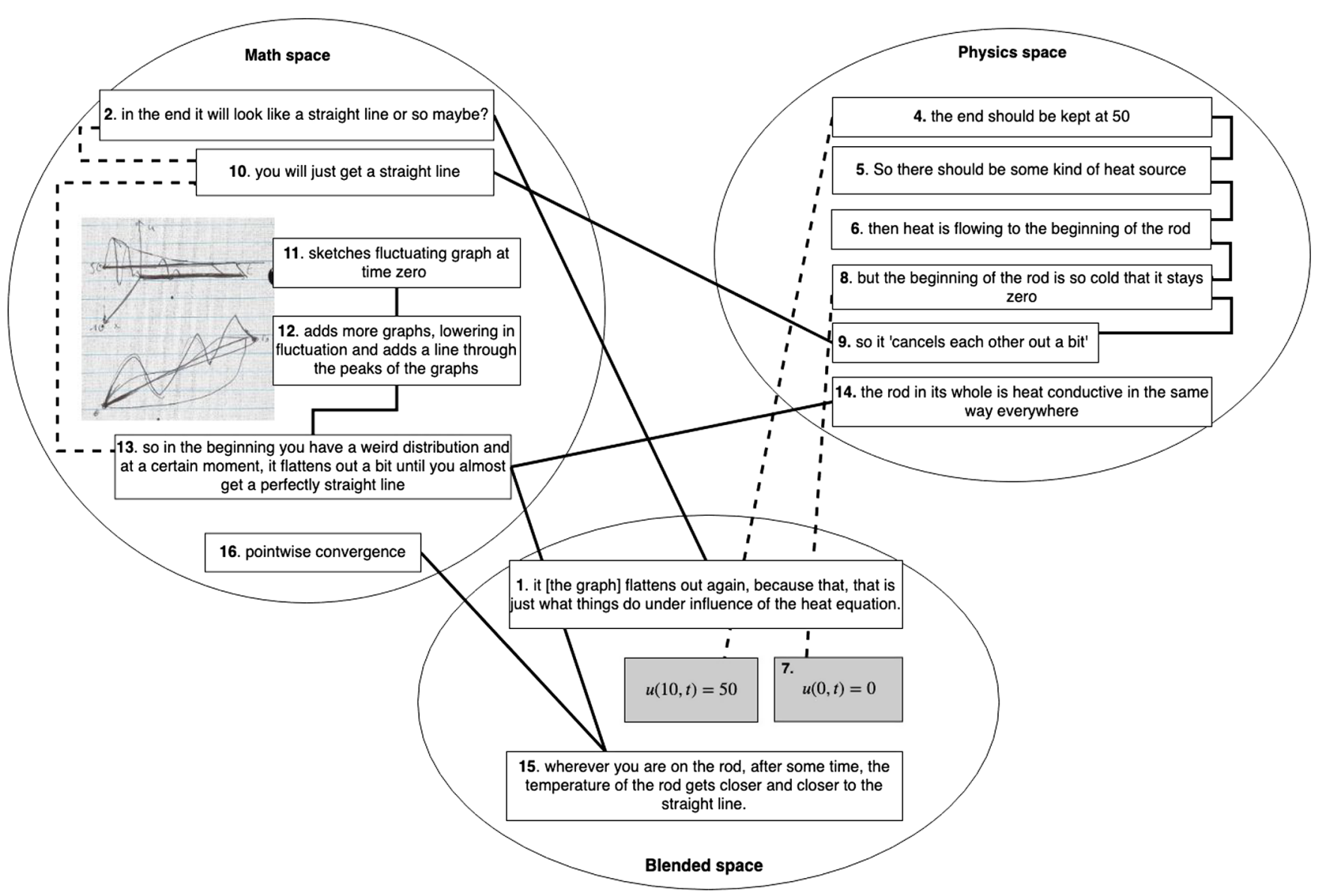

FIG. 10. DBD 2: David and Evan's answer to question B-II.

temperature is fixed at zero degrees (7, gray box, and $\mathbf{8}$ ). Similarly as in elements 3 and 4 in the previous paragraph, element 7 here is placed in the blended space and implicitly connected to its physical interpretation (8). The students add in element 9 that "it cancels each other out a bit," which is also placed in the physics space as it is a continuation of their physical line of reasoning. We conjecture that this can be interpreted as follows: heat flowing in from one side flows out from the other. However, we cannot be certain that this interpretation is correct.

The students conclude this line of reasoning by repeating the statement that the graph will evolve to a straight line (10), which is placed in the mathematics space because it discusses the shape of the graph in a mathematical way. Element 10 is explicitly connected to element 9 as it follows directly from their reasoning in the physics space, and implicitly connected to element 2 because it confirms what they were thinking before.

The students continue their discussion by repeating the values of the function at the boundaries, which were discussed already in DBD 1. Therefore, these are not selected as new elements.
Evan 23:58 Yes, at $x$ is ten it is held at 50, so that is this high (puts a dot at these coordinates in the axes). Yeah maybe that's not really drawn well. But the thing is then, in the beginning you have a weird distribution [sketches fluctuating graph in coordinate system (11)], and at a certain moment, it flattens out a bit [adds more graphs, lowering in fluctuation and adds a line through the peaks of the graphs (12)] until you almost get a perfectly straight line (13).

David 24:33 Yes, yes

Evan 24:34 Cause, yeah, if it wouldn't be a straight line, then it should be something else and I cannot imagine what it would, would be then. Because the rod in its whole is heat conductive in the same way everywhere (14). So, if this side is held perfectly at zero and that side is perfectly held at 50 (adds " 0 " to the starting point of the three graphs and "50" to the end point of the three graphs) and everything in between is heat conductive in the same way, it doesn't seem to me that it would be something else than a straight line...

In this excerpt, David uses a graph to explain his thinking. He starts by sketching an initial graph (11) and then adds graphs at later times with lower fluctuations (12). 
He does this in a three-dimensional set of axis and also in front view. He describes how this shape evolves towards a straight line (13). This set of elements is explicitly connected and all placed in the mathematics space as they are referring to the graphical representation in a nonphysical way. Element 13 is also connected to element 10 with a dotted line because it builds on their earlier idea that the shape will evolve to a straight line.

Then, Evan adds that the heat conductivity of the rod is the same everywhere (14, physics space), which he considers a reason for the graph to evolve towards a straight line in the end. Therefore element 14 is connected to element 12. Note that in the last sentence, the student revisits the cycle of ideas 4-8-14-10, which are all in some way connected in the diagram, as a way of concluding this phase of reasoning.

The students reread interview question 2 and continue:

David 25:41 Well, I just think that the, the closer you are to position zero, the ehm... Ah wait, no, wait... It can vary, the temperature, but the closer it is going to get to a value on the straight line...

Evan 26:00 Hmhm.
David 26:00 after some time. And that, yeah, that at, wherever you are on the rod, that after some time, the temperature of the rod gets closer and closer to the straight line (15).

Evan 26:18 Yeah. Pointwise convergence (16).

David 26:19 Yes.

The students build on the mathematical idea of the converging graph (13) and also explicitly refer to the physical context in element 15, therefore it is placed in the blended space. This is directly connected to the mathematical concept of pointwise convergence (16).

\section{Question III}

At this point in the interview the students move on to question III, where they start to think more deeply about the meaning of heat flow, and in particular its mathematical definition. The DBD for this part, DBD 3, can be found in Fig. 11.

Evan 26:37 Evolution of the values of temperature. Yes and, for the heat flow I don't really have a clue Ehm... I don't even know the definition of heat flow.

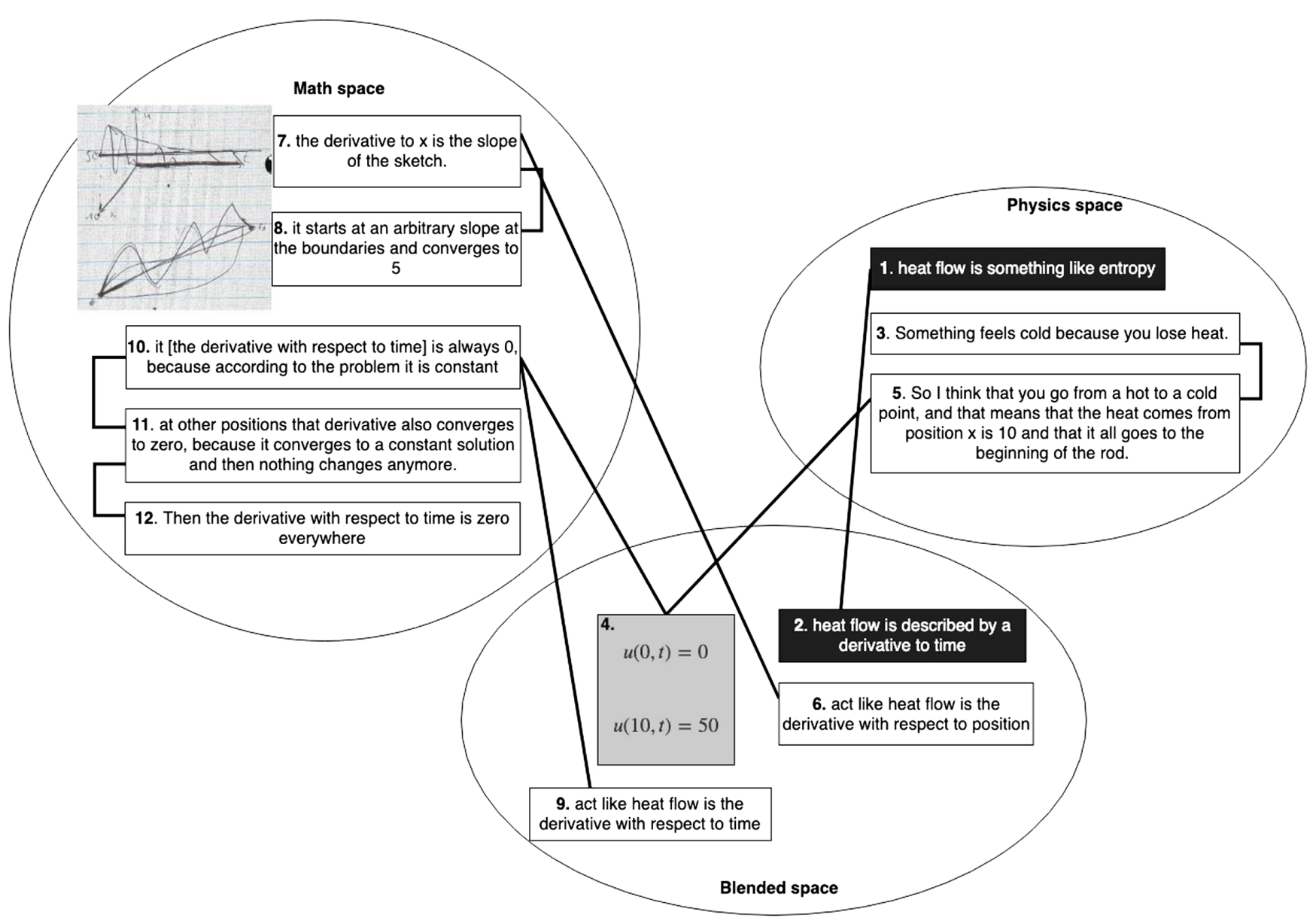

FIG. 11. DBD 3: David and Evan's answer to question B-III. 
Interviewer 26:45 Yes, so how does heat flow in the system?

Evan 26:48 In the whole system? Or at the boundary points?

Interviewer 26:50 The question is to discuss it at the boundary points.

Evan 26:53 Okay. Ehm... and the heat flow, that is the derivative to the space dimension? Of the heat? Or the derivative to time?

David 27:05 Eh, wait... Entropy...

Interviewer 27:06 That's a good question.

Evan 27:08 Thanks.

David 27:08 I think it is entropy (1), so derivative to time (2) if I'm not mistaken...

Evan 27:18 Heat flow, yeah, I've never really understood at the exam what he meant by heat flow. Like yeah... The funny thing is that both options could be defended in some way, right?

David 27:31 Yes. Ehm.

Evan 27:33 Yes if you, if we don't have the definition, we cannot answer the question, actually, right?

David 27:38 If I'm not mistaken, ehm, heat flow is something like entropy (1).

Evan 27:45 I don't have a clue what entropy is (laughs). David 27:45 Yeah I don't really know it myself but...

Interviewer 27:50 But try to use you, ehm, your intuition and daily insight, to try to understand heat.

David 28:01 Something feels cold because you lose heat (3). So I think that you go from a hot to a cold point [Referring to the boundary conditions (4)], and that means that the heat comes from position $x$ is 10 and that... And that all goes to the beginning of the rod (5).

As the students start thinking about the meaning of heat flow, they acknowledge that they do not know the definition of heat flow. David proposes that heat flow is something like entropy (1, physics space), which makes him think heat flow should be described by a derivative with respect to time (2). This is placed in the blended space because it ties a mathematical description to the physical concept of heat flow. Again, the students have problems understanding this because neither of them really knows what entropy means. It is unclear how the students decide from the concept of entropy that the description of heat flow should be the derivative of temperature with respect to time. Elements 1 and 2 are both colored black in the diagram to highlight that these elements are incorrect. In the end, and only after having been prompted by the interviewer, David adds an interpretation of heat flow coming from his daily-life experience (3, physics space) and he adds what this implies for the system (5, physics space) based on the boundary conditions from the problem statement (4, blended space).

In what follows, the students consider two possible mathematical definitions for heat flow. They start by considering the derivative of temperature with respect to position as the heat flow:

Evan 30:27 Okay. Hm. They could have defined heat flow for me in the course.. Discuss what the boundary conditions mean for the evolution of values of temperature.. You know what? We just do it for both and then... we still win time then.

David 30:47 Hmhm.

Evan 30:47 Let's just for now just act like heat flow is just the derivative to position of $u(6)$.

David 30:56 Yes.

Evan 30:57 Okay, well, then we have to discuss that, will be fine. Discuss what the boundary conditions mean for the evolution of the values of temperature/concentration, yes just temperature, and heat flow. Okay so the boundary conditions are, that here it stays zero always and here 50. David 31:14 Yes.

Evan 31:14 and what does that mean for the derivative to $x$. Well, the derivative to $x$ is just the slope of the sketch (7).

David 31:22 Hmhm.

Evan 31:23 Ehm, That doesn't mean a lot, that, at least... Yes, Yes, it does mean something, that ehm, that one starts at an arbitrary slope at the boundaries, doesn't matter what, because the beginning... a weird initial distribution [double marks the first drawn graph in previously sketched image (fourth graphs total)] and that converges to... that 50 , minus zero over 10 minus zero, so yeah 5 (8), yes. Five.

David 31:43 Yes, five.

Evan 31:43 The sketch is not perfect, but that converges to five, okay.

The students investigate the hypothesis that the derivative of temperature with respect to position represents heat flow (6). This is placed in the blended space because it ties a mathematical description to the physical concept of heat flow. Then they connect mathematical meaning to it (7 and 8), based on the graphs from before and show good mathematical insight: the slope with respect to $\mathrm{x}$ can initially have any value, but will converge to 5 . However, there are no lines going to the physics space, indicating that the connection to the physical meaning of heat flow is missing.

Secondly, the students consider the derivative of temperature with respect to time to be the heat flow:

Evan 31:43 Well, great result. And now to time. Eh, how should we look at this. Imagine that heat flow is the derivative of $u$ to time in a certain point at a certain time (9)... Eh, that is a bit harder to imagine, right. Ehm, derivative to time.

David 32:11 Ah yeah wait...

Evan 32:13 That means that it, ah yeah, yes, (referring to the boundary conditions) that is just in, in,... in 10 it is always 50 and in 0 it is always $0 .$. (repetition of 4) 
David 32:19 Hmhm.

Evan 32:17 because there it is, so there it (the derivative with respect to time) is constant according to the problem (10).

David 32:20 Yes.

Evan 32:22 And at other positions that derivative also converges to zero. Because at a certain moment, it converges to a constant solution and there nothing changes anymore (11), so...

David 32:30 Yes, it just, yes.

Evan 32:36 Yes, nothing changes anymore. That means

that the derivative to $t . .$. to time is zero (12).

David 32:40 Hmhm.

Evan 32:40 Yes, okay, we also have a nice resul... we have a result. Okay, succeeded after all.

In this fragment, the students attempt to consider the derivative of temperature with respect to time as representing heat flow (9, blended space). They use the information from the boundary conditions in the problem statement (4, gray box) to argue that the derivative with respect to time should be zero at the boundaries (10). Next, they extend their reasoning to the rest of the system (11) without any clear argumentation and conclude that the derivative with respect to time should converge to zero everywhere (12). All of this reasoning is situated in the mathematics space and explicitly connected. Again, the students never refer to the physical process this represents.

The interviewer then attempts to make them choose:

Interviewer 32:43 And can you try to choose one of the two now to be the heat flow?

Evan 32:48 Yes, intuitively I find this most sensible, that (the derivative of $u$ with respect to $x$ ) is the heat flow. Interviewer 32:51 So the one to $x$ ?

Evan 32:54 It doesn't really matter I think, what it, which of the two it is, it is just a name...

Interviewer 32:58 Yes, but the question is 'discuss heat flow,' so if you are going to say...

Evan 33:01 Yes, that is kind of true...

The students cannot decide between the two options, and even suggest that it does not matter. Using the blending diagram in Fig. 11 we can see that the problem here is the lack of elements in the physics space and connections to the physics space. They have some (limited) physical understanding of heat flow (element 3), but they fail to connect it to their two mathematical options.

\section{B. Case 2}

As a second case, we discuss the answers of students Fin and Glen to interview task A (see Fig. 6). This case was selected because it shows how a DBD can help in visualizing and interpreting the line of reasoning of the students. We do not discuss their answer to task A-I because the features of that DBD are very similar to the earlier discussed DBD 1. Here, we discuss the answers to A-II and III. Because the students answered these two questions in an integrated way, this resulted in one DBD instead of two. Figure 12 shows DBD 4.

The interviewer reads question II of interview task A aloud and the students start their answer:

Fin 10:55 Yeah, [referring to the boundary conditions (1)] the temperature on both sides stays constant so, over time (2) and...

Glen 11:02 and the heat flow is just the derivative of temperature to position (3).

Fin 11:07 Yes, and that means that eventually the whole rod will just get a temperature of zero. After, yeah, not a lot of time (4).

Glen 11:11 Okay yeah. It's very badly isolated.

Fin 11:16 Yes, even not isolated. (5)

The students start by stating a physical interpretation of what happens at the boundaries, which is probably based on the equations of the boundary conditions (1, gray box, and 2, which are implicitly connected). Thereafter, Glen mentions an interesting element: "heat flow is just the derivative to position," which is placed in the blended space (3). In this specific case, the numbering points out to a reader that this element is mentioned in the very beginning of their reasoning, without a lot of ideas building up to it. Fin continues by mentioning that the whole rod will evolve to a temperature of zero (4). Note that elements 2 and 4 actually follow each other in one sentence, while student Glen interrupted with element 3. Therefore, we connect elements 2 and 4 with a dotted line, as they are probably part of one line of reasoning. Lastly, the students conclude that the rod must be badly or even not isolated (5). It seems that this statement is based on element 4. Therefore, we connect elements 4 and 5 with a dotted line.

The interviewer summarizes the two questions to make the students continue:

Interviewer 11:21 Yes, so, the second question is, during the process and the third question is after a very long time, so you can actually discuss those two at the same time. Glen 11:26 Ah, okay.(talking at the same time)

Fin 11:28 Ah, yes, yes.

Glen 11:30 Imagine you have a sine wave (6) [this refers to a possible initial condition (7)], then it will also...

Fin 11:32 For example yeah...

Interviewer 11:35 You can sketch if you'd like.

Fin 11:36 Yes.

Glen 11:36 Ah, okay, ah yeah, okay. The difference with the sine is just that instead of here (they refer to the horizontal line at average height on the graph), you will get a line here [adds a horizontal line at height zero on the graph (8)] 


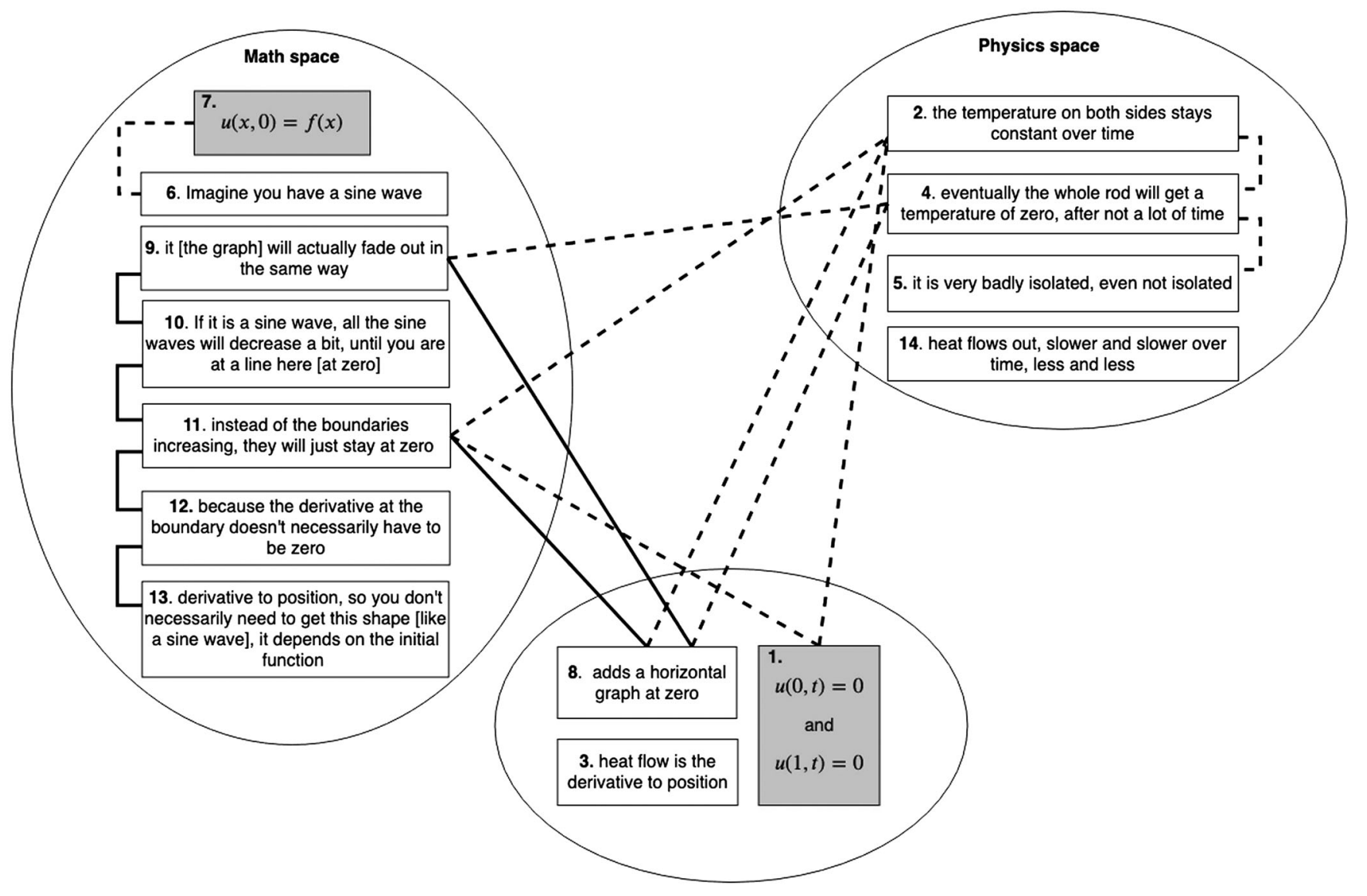

FIG. 12. DBD 4: Fin and Glen's answer to tasks A-II and III.

Fin 11:45 Yes, yes.

Glen 11:46 And so, but it will actually fade out in the same way (9), like, cause it will start here doing just... Like all those sine waves will, like... If it is a sine wave, all the sine waves will decrease a bit, until you are at a line here (at zero) (10). That is like...

Fin 11:58 So instead of the boundaries increasing, they will just stay at zero (11). Yes, because they fade out.

Glen proposes to start from a sine-shaped function (6, mathematics space). This probably refers to the shape of the initial condition that they used in the previous problem, which they also used in their answer to A-I. The students actively compare this problem with the previous one, where they were given an isolated system where the temperature distribution was initially shaped like the graph in Fig. 13. In that problem, the students concluded that the solution is a straight line at the average height. Now, the students draw a horizontal graph at height zero on that same set of axes (8). This is placed in the blended space because it is a graphical representation of what they stated earlier in element 4 from the physics space. The students expand their comparison with the previous problem and start a mathematical line of reasoning. They mention that the graph will fade out similarly (9, mathematics space), which is connected to the graph they sketched in element 8 . This builds on the previously mentioned idea that the rod will evolve to a uniform temperature of zero (implicit connection between 4 and 9). Element 9 is categorized as being mathematical because it discusses the evolution of the graph without

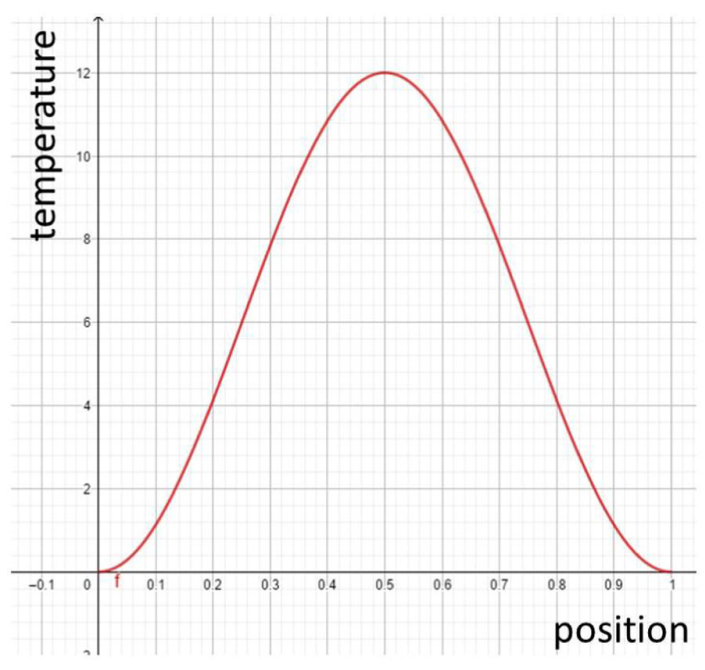

FIG. 13. IC given in the previous problem (which is not discussed in this paper). It has the shape of a shifted sine curve. 
referring to the physical process. This line of reasoning forms the triangle 4-8-9. Then Glen continues the comparison and states that If it is a sine wave, all the sine waves will decrease a bit, until you are at a line here (10, mathematics space). He seems to use the initial condition from the previous problem and combines this with the earlier mentioned idea of the decreasing graph converging to zero. To continue, the students mention the graphical representations of the boundary conditions (11, mathematics space), which is connected to previously mentioned elements to form triangles 11-1-2 and 11-8-2.

The students continue by discussing a partial derivative at the boundary.

Glen 12:05 Ah yeah, because the derivative doesn't necessarily have to be equal to zero (12).

Fin 12:09 Eh, Yes.

Glen 12:10 Ah yes, but it does if we have a sine wave by coincidence.

Fin 12:14 Yes.

Interviewer 12:15 Wait, the derivative doesn't have to be equal to zero? What do you mean by that?

Glen 12:18 the derivative at the boundary.

Fin 12:21 And to time or position?

Glen 12:23 The?

Fin 12:23 Time or position?

Glen 12:24 Position, so like, you don't necessarily need to get this shape (13).

Fin 12:27 Ah yeah, true.

Glen 12:30 But it depends on the initial function (continuation of 13).

Interviewer 12:32 Yes.

Fin 12:34 I think so?

Glen starts by adding that "the derivative does not have to be zero" (12). We include a line to element 11 because the student uses element 12 to justify element 11 . The interviewer asks the students to explain what they mean by that, which causes Fin to ask "to time or to position?" We know from previous work that students have difficulties with different aspects of taking the derivative of a function with two variables, so this is an important question (ref study 1). In element 13, the student specifies that he means the partial derivative with respect to position, but he adds "you don't necessarily need to get this shape, it depends on the initial function." It is not completely clear what the student means by this, but a possible interpretation is that he is referring to the slope at the boundaries and how the initial function determines that. As the derivative with respect to position does not have to be zero by default in this case, this means that the slope should not necessarily be zero and therefore, the tangent line should not necessarily be horizontal. Even though the students reason about the derivative with respect to position, we do not connect it with element 3 , because at no point in this reasoning they refer to heat flow or any other physical meaning.
The interviewer finishes by asking them to repeat and summarize their answer concerning heat flow at the boundaries.

Interviewer 12:35 So can you specify again how the heat fl... So you said it already for the temperature, but what can you say about the heat flow at the boundaries? Throughout the process?

Glen 12:44 It is flowing out, and I think slower and slower.

Fin 12:49 Yeah, eh, yes.

Glen 12:52 Always less and less. Ah yes yes, less and less heat (14), so less heat will flow out.

This results in the students adding some more physical ideas (14). This element is not connected to anything in the diagram because the students do not explicitly state the reason for the heat flow being "slower and slower."

In conclusion, at first sight these students seem to have a good and well-connected understanding. They constantly move between spaces and actively build on and connect the different parts of their reasoning. However, after mapping the transcript to a blending diagram, we see that the critical element 3 heat flow is the derivative to position is not explicitly connected to anything. This means that we cannot be sure that the students have a complete understanding of all the elements they use and how they are connected.

\section{DISCUSSION}

In this paper, we use the conceptual blending framework as a lens to study student thinking while combining mathematics and physics in the context of phenomena described by the heat equation. We propose the dynamic blending diagram as a way to analyze student reasoning. The DBD differs from blending diagrams earlier presented in PER by adding time stamps and implicit and explicit connections at element level so as to capture the dynamics of the reasoning. We used data from an interview study and selected two cases to illustrate the construction of a DBD.

Because of our focus on the interplay of mathematics and physics, we chose to define a mathematics and a physics space as input spaces. We explicitly formulated a characterization of these input spaces and the blended space, a step that is not explicitly mentioned in the earlier work in PER and MER discussed in Sec. IC. In particular, we consider equations and graphs as blended elements, i.e., as elements that combine mathematics and physics, when they are used to carry the meaning of the physical concepts they represent [20]. A similar perspective on the role of graphs is seen in the work of Gerson and Walter [22]. In their work, the abstract graphs are positioned in one of the input spaces, i.e., the inflow and outflow space, but once this graph is used to explicitly discuss the water in the reservoir, it becomes a blended element. 
Using our definitions for the different spaces (Table I), equations and graphs can typically be in two spaces: mathematics or B. It is possible that students first use an equation in a mathematical way (e.g., element 2 in DBD 1) and then later on in their reasoning give a physical interpretation to it (e.g., element 4 in DBD 1). From then on, element 2 has become a blended element, because it is an equation to which students attach physical meaning. Therefore, in the final DBD, we place element 2 in the blended space. The fact that the element moved is implicitly covered by the numbering. So when an equation has a chronological number that comes before its physical interpretation, it has moved throughout the process.

In our data, the boundary conditions are provided in the task and the students are asked to give and explain their physical interpretation. The blending would be of a different nature if the students would have been asked to construct these boundary conditions themselves based on a description of a physical situation. However, the boundary conditions would end up in the blended space in both cases most of the time. The representation in the DBD works for both types of data because of the color code and the numbering. The color gray is used to mark elements that were given in the problem statement. In our data, we see that the boundary conditions are invoked early on in the reasoning. In the case where students would have to construct the boundary conditions themselves, we would expect the boundary conditions almost always at the end, chronologically.

The structure of our DBDs and the characterization of the spaces result partly from an important choice in the design of the diagrams: only elements that are explicitly mentioned by the students are used in the DBD and copied as literally as possible. This contrasts to other studies in PER (i.e., Refs. $[4,12,13,20]$ ), where the input spaces generally contained the concepts and ideas from the different fields that were needed to formulate the student's explanation, even if the students did not necessarily explicitly mention all of them. In these blending diagrams, the authors' interpretations of student's explanations were crucial to complete the input spaces. In our diagrams, the role of the researchers' interpretations is minimized as much as possible. The authors do not add implicit elements in the analysis. Moreover, by using dotted lines, we show the reader that the connection is an interpretation.

As a consequence of this design choice, it is possible to have disconnected elements in the blended space that combine mathematics and physics ideas, but do not seem to originate from elements in the input spaces. From the data alone, there is no way of knowing if the students possessed all the necessary elements in the inputs to come to this blended insight. It is also possible to have a connection between a blended element and a physics element without having a link to the mathematics space. For instance, when the students connect a physical interpretation to an equation, this equation is placed in the blended space and connected to the interpretation in the physics space. Implicitly, the student probably used mathematical knowledge to read the equation. In the work of Schermerhorn and Thompson [20] this is where symbolic forms [21] are invoked to show what implicit mathematical ideas are being used to construct or interpret equations. In our work, however, the blending does not acknowledge implicit ideas and we do not add interpretations from the researcher's perspective, so this mathematical knowledge stays implicit.

Whereas in earlier work in PER the focus is on the resulting blended space, we argue that in our visualization in a DBD, the blended space does not give a full overview of the product of the blending process. The blending has to be interpreted in a broader way than just the elements in the blended space. The blended space contains elements that combine mathematics and physics ideas, and graphs and equations that carry physical meaning. However, these always have to be interpreted in combination with the existing connections to the physics and mathematics spaces.

The main aim of constructing DBDs is of course to use it as a method to get more insight in student reasoning. The introduction of individual connections at element level enables us to judge the degree of integration in the blending diagrams. By numbering the elements, the reader can follow how the students move around between spaces throughout their reasoning. The combination of the individual connections and the numbering enables the reader to follow the line of reasoning of the students in the DBD.

In the case studies discussed in this paper, we observe some well-integrated networks in DBDs 1,2, and 4. These DBDs typically contain triangles that connect elements in all three spaces. In DBDs 1 and 2 the students actively move between all spaces. In DBD 4, a different type of process is visible. The students initially show mostly physical reasoning (1-5) before moving on to mostly mathematical reasoning (7-12) and only in the end they add another physics element which is not well connected to the rest of the reasoning. These mathematical and physical lines of reasoning are connected implicitly in several ways in the diagram. However, we also observe a poorly integrated network in DBD 3, with one-sided connections between the mathematics and the blended space, leaving the physics space somewhat isolated. In contrast to DBD 4, where the physics space was well developed and implicitly connected to the reasoning, the disconnection to the physics space in DBD 3 causes difficulties.

In addition to describing the level of integration, the DBD can also be used to diagnose and locate difficulties in student reasoning. For example, in DBD 3, the absence of connections between elements in the mathematics and physics space prevents the students from finding the relation between heat flow and its mathematical description. As a second example, in DBD 4, element 3, "heat flow 
is derivative to position," is not connected to the rest of the reasoning. The students make a statement about heat flow (13) and about the derivative to position not having to be equal to zero (11 and 12), but do not connect the elements to the initial idea which indicates incomplete understanding.

By using black colored boxes, we acknowledge that students sometimes use incorrect elements in their reasoning. In DBD 3, for example, this representation immediately shows that there is a difficulty situated around elements 1 and 2, where the students connect heat flow to entropy and to a derivative with respect to time.

Conceptual blending has the potential to be a powerful framework to investigate the combination of mathematics and physics in student reasoning. We built on previous adaptations of the original framework in PER and MER and added several aspects that enable us to investigate the process of reasoning. However, not all constructs from the original work of Fauconnier and Turner have already been translated to the PER context.

The attentive reader has noticed that the generic space has no place in our DBDs. In our study we see the generic space as providing structure from inferred reasoning that depends on researcher interpretation. Since we limit our analysis to explicit statements and reasoning, we also omit a generic space in our blends. We do not distinguish between the different mechanisms to generate new meaning in the blended space (composition, completion, and elaboration). In the examples presented here, students combine ideas and concepts from mathematics and physics, which could be interpreted as composition. However, pinpointing where composition turns into completion and even elaboration appears to be difficult. Studies from PER and MER dealing with the interplay between mathematics and physics also do not mention the three distinct mechanisms.

\section{LIMITATIONS AND FUTURE RESEARCH}

A dynamic blending diagram provides specific details about students' reasoning processes and how they connect mathematics and physics ideas. This extensive analysis can also provide insight in difficulties in student reasoning and as such scaffold the design of effective teaching materials.
However, constructing a DBD is not obvious and is time consuming. Therefore, it is not suitable for quick analysis or directly as a teaching tool.

The structure of the DBD is closely related to the type of questions in the interview and the nature of the data we are using. The DBDs as defined and constructed in this paper are suitable to investigate qualitative and conceptual reasoning, but may be less suitable to visualize reasoning with a prominent role for calculations. Like in most other work in PER that studies the interplay between mathematics and physics through a conceptual blending lens, we describe the mechanism of blending on a rather general level: we do not distinguish between the three mechanisms of blending as they are described in the original framework. However, it is an interesting challenge to better understand the differences between these mechanisms in the context of mathematics and physics. Our case studies use the context of the heat equation, but the analysis method is applicable to other contexts in which students are expected to blend mathematics and physics. By extension, it should also work for topics in which any two subjects are combined, e.g., chemistry and biology or mathematics and economics. However, describing other contexts requires again a careful definition of the input spaces.

The aim of this paper is to propose the method of constructing a dynamic blending diagram. Content-specific analysis using DBDs will follow in a subsequent paper. Combined with our earlier work on student difficulties with boundary conditions, this will scaffold the development of a teaching intervention in order to improve the learning process of boundary conditions in the context of the heat equation.

\section{ACKNOWLEDGMENTS}

We want to thank all participating students for their voluntarily participation to our interviews. We also thank Dr. Jan Sermeus for reading our manuscript and sharing his constructive thoughts with us. This material is based upon work partially supported by the National Science Foundation under Grants No. PHY-1405726 and No. PHY1912087.
[1] B. P. Schermerhorn and J. R. Thompson, Physics students' construction of differential length vectors in an unconventional spherical coordinate system, Phys. Rev. Phys. Educ. Res. 15, 010111 (2019).

[2] T. I. Smith, J. R. Thompson, and D. B. Mountcastle, Student understanding of taylor series expansions in statistical mechanics, Phys. Rev. ST Phys. Educ. Res. 9, 020110 (2013).

[3] J. Wagner, Students' obstacles and resistance to Riemann sum interpretations of the definite integral, in Proceedings of the 19th annual Conference on Research in Undergraduate Mathematics Education, edited by T. Fukawa-Connelly, 
N. Engelke Infante, M. Wawro, and S. Brown (MAA, Pittsburgh, 2016), pp. 1385-1392.

[4] D. Hu and N.S. Rebello, Using conceptual blending to describe how students use mathematical integrals in physics, Phys. Rev. ST Phys. Educ. Res. 9, 020118 (2013).

[5] B. R. Wilcox, M. D. Caballero, D. A. Rehn, and S. J. Pollock, Analytic framework for students' use of mathematics in upper-division physics, Phys. Rev. ST Phys. Educ. Res. 9, 020119 (2013).

[6] J. Guisasola, J. Almundi, J. Salinas, K. Zuza, and M. Ceberio, The Gauss and Ampere laws: Different laws but similar difficulties for student, Eur. J. Phys. 29, 1005 (2008).

[7] L. Doughty, E. Mcloughlin, P. V. Kampen, L. Doughty, E. Mcloughlin, and P. V. Kampen, What integration cues, and what cues integration in intermediate electromagnetism, Am. J. Phys. 82, 1093 (2014).

[8] L. Bollen, P. V. Kampen, and M. D. Cock, Students' difficulties with vector calculus in electrodynamics, Phys. Rev. ST Phys. Educ. Res. 11, 020129 (2015).

[9] R. Pepper, S. Chasteen, S. Pollock, and K. Perkins, Observations on student difficulties with mathematics in upper-division electricity and magnetism, Phys. Rev. ST Phys. Educ. Res. 8, 010111 (2012).

[10] E. F. Redish and E. Kuo, Language of physics, language of math: Disciplinary culture and dynamic epistemology, Sci. Educ. 24, 561 (2015).

[11] G. Fauconnier and M. Turner, Conceptual blending, form and meaning, Recherches en communication 19, 57 (2003).

[12] T. J. Bing and E. F. Redish, The cognitive blending of mathematics and physics knowledge, AIP Conf. Proc. 883, 26 (2007).

[13] L. Bollen, P. van Kampen, C. Baily, and M. De Cock, Qualitative investigation into students' use of divergence and curl in electromagnetism, Phys. Rev. Phys. Educ. Res. 12, 020134 (2016).
[14] T. Huynh and E. C. Sayre, Blending of conceptual physics and mathematical signs, arXiv:1909.11618.

[15] M. L. Boas, Mathematical Methods in the Physical Sciences (Wiley, New York, 2006).

[16] S. Van den Eynde, J. Deprez, M. Goedhart, and M. De Cock, Undergraduate student's difficulties with boundary conditions for the diffusion equation, Int. J. Math. Educ. Sci. Technol. (to be published).

[17] S. J. Farlow, Partial Differential Equations for Scientists and Engineers (Courier Corporation, New York, 1993).

[18] G. Fauconnier and M. Turner, Conceptual integration networks, Cogn. Sci. 22, 133 (1998).

[19] O. Uhden, R. Karam, M. Pietrocola, and G. Pospiech, Modelling mathematical reasoning in physics education, Sci. Educ. 21, 485 (2012).

[20] B. Schermerhorn, Investigating student understanding of vector calculus in upper-division electricity and magnetism: Construction and determination of differential element in non-Cartesian coordinate systems, Ph.D. thesis, University of Maine, 2014.

[21] B. L. Sherin, How Students Understand Physics Equations, Cognit. Instr. 19, 479 (2001).

[22] H. Gerson and J. Walter, How blending illuminates understandings of calculus, in Electronic Proceedings for the Eleventh Special Interest Group of the Mathematical Association of America on Research in Undergraduate Mathematics (2008).

[23] M. Zandieh, K. H. Roh, and J. Knapp, Conceptual blending: Student reasoning when proving "conditional implies conditional" statements, J. Math. Behav. 33, 209 (2014).

[24] C. Megowan and M. J. Zandieh, A case of distributed cognition (or, many heads make light work), in Proceedings of the 27th annual meeting of the North American Chapter of the International Group for the Psychology of Mathematics Education (2005), http://www.pmena.org/ proceedings/. 